\title{
Multiple nodular form of localized pigmented villonodular synovitis in the infrapatellar fat pad: A case report
}

\author{
Sungwook Choi, Sang-Rim Kim, Jun-Yeong Seo, In-Seok Son, Yong suk Kwon, and Yong-Geun Park* \\ Department of Orthopaedic Surgery, Jeju National University Hospital, Jeju National University School of Medicine, Jeju, Korea
}

\begin{abstract}
Pigmented villonodular synovitis (PVNS) is a rare, benign, proliferative disease of the synovial membrane of joints, tendon sheaths, and bursas. It usually presents in two forms, diffuse and localized. A 35-year-old male, since adolescence, presented left knee pain with palpable, multiple nodular tumorous lesions in the infrapatellar fat pad. An arthroscopic excisional biopsy was performed. Multiple brown-colored pedunculated synovial nodules were limited to the infrapatellar fat pad area. The histology of the resected specimens showed proliferation of mononuclear histiocytes with multinucleated giant cells and hyperplasia of the synovium, diagnosed as PVNS. LPVNS is usually a single mass with a pedunculated or, less frequently, a sessile appearance. This report describes a rare case of a multiple nodular form of LPVNS, localized at the infrapatellar fat pad. This case highlights the need to consider LPVNS one of the causes of vague and long-lasting knee pain.
\end{abstract}

\begin{abstract}
Abbreviations: PVNS: Pigmented Villonodular Synovitis; DPVNS: Diffuse Pigmented Villonodular Synovitis; LPVNS: Localized Pigmented Villonodular Synovitis; MRI: Magnetic Resonance Imaging

\section{Introduction}

Pigmented villonodular synovitis (PVNS), also known as pigmented villonodular bursitis and pigmented villonodular tenosynovitis, is a benign, proliferative disease that occurs at the synovial membrane of joints, bursas, and tendon sheaths. There are two distinct forms: diffuse (DPVNS) and localized (LPVNS) pigmented villonodular synovitis [1]. The diffuse form affects the whole synovium of the joint, while the localized form is characterized by focal involvement of the synovium [2]. The localized form is usually a single pedunculated mass or, less frequently, two or three nodules. Finding multiple nodules in LPVNS is extremely rare. To the best of our knowledge, there are only two reported cases [3]. Here, we describe a case of the multiple nodular form of LPVNS in the knee joint. It should be included as an atypical form of LPVNS in the differential diagnosis of knee problems.
\end{abstract}

\section{Case report}

A 35-year-old male visited our hospital with left knee pain and a palpable mass which developed since adolescence. Physical examination revealed neither effusion nor restriction of knee motion. Modest swelling was palpable over the infrapatellar area of the left knee versus the right side. He had tenderness around the patella tendon and tibial tuberosity. Laboratory studies revealed no abnormal findings. Plain radiographs showed a slightly increased radiopacity in the infrapatellar area and soft tissue swelling versus the contralateral side (Figure 1). Magnetic resonance imaging (MRI) showed multiple well-demarcated masses, with intermediate to low signal intensity on T1- and T2-weighted images, surrounded by fluid signals in the anterior compartment of the knee joint (Figure 2). He underwent an arthroscopic excisional biopsy to remove the mass. Multiple brown-colored pedunculated nodules were found; they were limited to the infrapatellar fat pad (Figure 3). No other lesion was found throughout the knee joint. A pathology examination revealed the nodules surrounded by fibrous septa and foci of hemosiderin-laden macrophages and the proliferation of polyhedral mononuclear histiocytic cells, some of which had formed multinucleated giant cells. There was mild hyperplasia of the synovium, with focal layers of fibrin consistent with pigmented PVNS (Figure 4). Three years later, he had neither pain nor a mass on palpation over the lesion. On follow-up MRI, no sign of recurrence was noted (Figure 5).

\section{Discussion}

The incidence of PVNS is $\sim 1.8$ cases per million people [4]. The etiology of PVNS is still uncertain: chronic inflammation, trauma, hemarthrosis, neoplastic origin, and chromosomal disorders have been

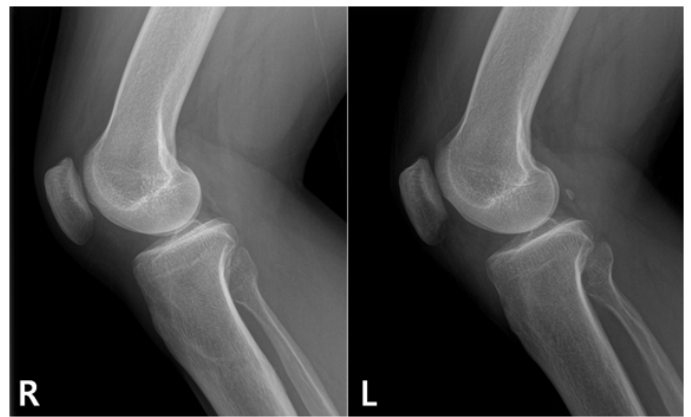

Figure 1. Lateral radiographs of the right knee (R) and left knee (L). The infrapatellar soft tissue is slightly more radiopaque in the left knee versus the right. However, it is difficult to distinguish them at a glance.

Correspondence to: Yong-Geun Park, Department of Orthopedic Surgery, Jeju National University Hospital, Jeju National University School of Medicine, Aran 13gil 15, Jeju 690-767, South Korea, Tel: +82-64-754-8116; Fax: +82-64-7171131; E-mail: pyk184@hanmail.net

Key words: pigmented villonodular synovitis, diffuse, localized, infrapatellar fat pad, arthroscopy

Received: October 05, 2016; Accepted: October 26, 2016; Published: October 29, 2016 

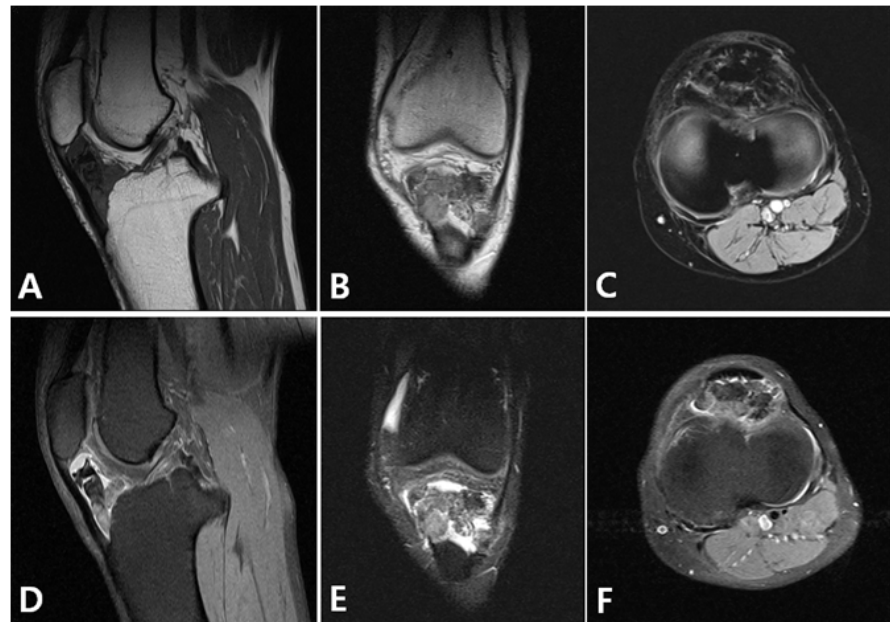

Figure 2. Preoperative MRI of the left knee shows multiple well-demarcated heterogeneou masses, with intermediate to low signal intensity on T1- and T2-weighted images, surrounded by a fluid signal in the anterior compartment of the knee joint (A. T1-weighted sagittal, B. T1-weighted coronal, C. T1-weighted axial, D. T2-weighted sagittal, E. T2weighted coronal, F. T2-weighted axial).

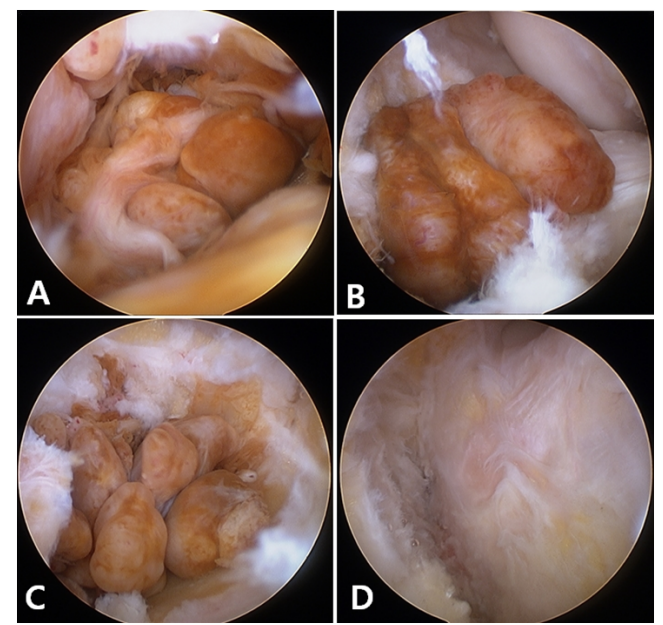

Figure 3. Arthroscopic view from an anterolateral portal showing several pedunculated LPVNS (A, B) and multiple nodules clustered in the infrapatellar fat pad area (C). D. Image after complete resection of LPVS.

considered [5]. The most widely accepted theories implicate a chronic inflammatory process or a benign neoplasia [6]. PVNS usually involves monoarticular joints: commonly, the knee joint. However, cases in the hip, ankle, shoulder, elbow, and even the temporomandibular joint have also been reported [4]. PVNS is usually diagnosed in patients between the third and fourth decades. It does not seem to have any gender predisposition [4]. The clinical presentation is diverse, and the diagnosis is often delayed because patients are usually asymptomatic. The most effective diagnostic modality is MRI [7]. Hemosiderin deposition in PVNS presents a low signal on T1- and T2-weighted images. Areas of bright signal on T2-weighted images may be present due to abnormal synovial membranes and joint fluid in the synovial membrane [8].

There are two types of PVNS: diffuse and localized [9]. Histological findings are similar but they are distinct disease entities for prognosis and treatment. The diffuse type, comprising $75 \%$ of PVNS cases, involves most of the synovium with accompanying joint effusion [10]. However, the localized form arises from certain parts of the synovium and resides within joints in a nodular form. The localized form is usually a single

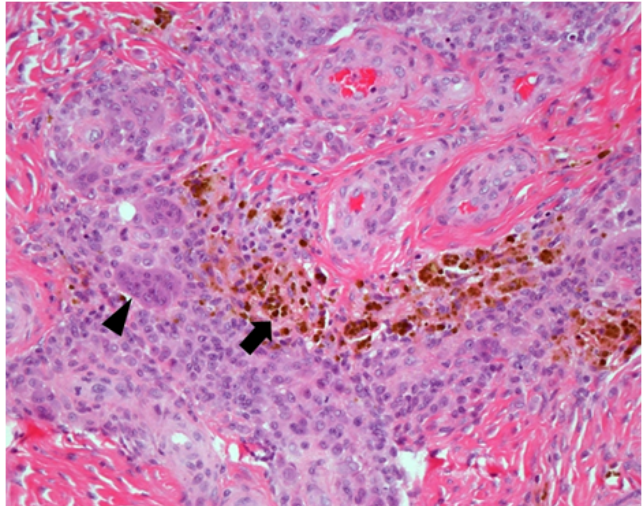

Figure 4. Pathological findings show the nodules surrounded by fibrous septa and foci of hemosiderin-laden macrophages (arrow), and infiltration of polymorphic inflammatory cells with mononuclear histiocytes, some of which were forming multinucleated giant cells (arrowhead). (hematoxylin and eosin staining: original magnification, $\times 200$ ).
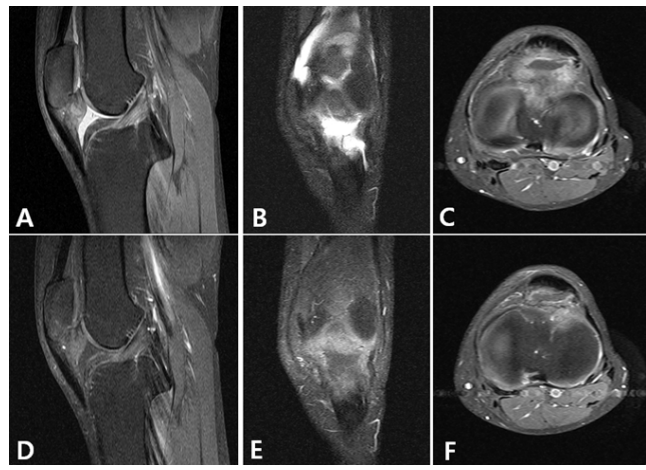

Figure 5. Follow-up enhanced MRI at 3 years after arthroscopic resection showed no recurrence of LPVNS in the whole knee joint. (A. T2-weighted proton density $<$ T2W PD $>$ sagittal, B. T2W PD coronal, C. T2W PD axial, D. Enhanced T1-weighted $<$ E-T1W $>$ sagittal, E. E-T1W coronal, F. E-T1W axial)

mass of pedunculated or, less frequently, sessile appearance that is yellow-brown in color. However, two or three multilobulated localized masses [11], and a mixed form, with a prominent mass and diffuse synovitis, representing a transition between DPVNS and LPVNS, have also been reported [12]. In our case, the multiple nodular form of PVNS was confined to the patellar fat pad, in a localized manner. Because the patient in this case had suffered from his symptoms for $\sim 20$ years, we assume that nodules had increased in number over a long time and finally presented as a multiple lesion. To the best of our knowledge, there is only one report of a multinodular form of LPVNS, referred to as clustered LPVNS [3].

Histologically, the circumscribed nodule is characterized by the active proliferation of fibroblastic and histiocytic elements, showing evident macrophagic activity with phagocytosis of abundant blood pigment (hemosiderin) and lipids. Those elements are intermingled with a variable number of multinucleated giant cells and with a certain number of inflammatory elements, predominantly lymphoplasmocytes [9]. Our case showed similar findings.

In treating PVNS, the purpose is to remove all abnormal synovial tissue, to prevent the risk of joint destruction, and to avoid local recurrence. For the knee joint, several options have been suggested, from observation to total knee arthroplasty, and including external beam radiation, radioactive synovectomy, surgical synovectomy, targeted therapy, immunotherapy, and combinations of them [13]. 
A recent systematic review reported no difference in local recurrence rates after open or arthroscopic surgery for LPVNS or DPVNS. However, a lower rate of postoperative complications was reported after arthroscopic surgery for DPVNS [14].

Recurrence rates of PVNS increase with time. Verspoor et al. [13] reported that $15 \%$ of recurrences occurred within the first year and $22 \%$ within 5 years in LPVNS [13]. They also reported that the 1 - and 5 -year recurrence-free survival rates for DPVNS were $69 \%$ and $32 \%$, respectively. In the present case, after arthroscopic resection, there was no recurrence at 3 years postoperatively.

In our case, the patient presented with long-lasting knee discomfort and mild swelling versus the contralateral knee. However, the range of motion was normal and no sign of instability, giving way, or blocking was seen. The patient has received just roentgenography, not special imaging, such as MRI, for $\sim 20$ years. Thus, we suspect that the LPVNS had grown slowly and finally became a multiple nodular form. Although there is no connection between LPVNS and DPVNS, LPVNS should be diagnosed and treated as soon as possible because early resection leads to a good prognosis. When there is vague and long lasting knee pain, LPVNS should be considered in the differential diagnosis and after the diagnosis is confirmed, early meticulous arthroscopic synovectomy is helpful for a good outcome.

\section{Acknowledgements}

All the authors have taken a significant and crucial part in the preparation of the article and that they have read and approved the final version.

\section{Conflicts of interest and source of funding}

Authors declare that there are no known conflicts of interest associated with this publication and there has been no significant financial support for this work that could have influenced its outcome.

\section{References}

1. Lee BJ, Yoo JE, Lee SH, Min KD (1998) Case Report Localized pigmented villonodular synovitis of the knee: arthroscopic treatment. Arthroscopy 14: 764-768.

2. Granowitz SP, Mankin HJ (1967) Localized pigmented villonodular synovitis of the knee. Report of five cases. J Bone Joint Surg Am 49: 122-128. [Crossref]

3. Kim RS, Kang JS, Jung JH, Park SW, Park IS, et al. (2005) Clustered localized pigmented villonodular synovitis. Arthroscopy 21: 761. [Crossref]

4. Myers BW, Masi AT, Feigenbaum SL (1980) Pigmented villonodular synovitis and tenosynovitis: a clinical epidemiologic study of 166 cases and literature review. Medicine 59: 223-238

5. Bouali H, Deppert EJ, Leventhal LJ, Reeves B, Pope T (2004) Pigmented villonodular synovitis: a disease in evolution. $J$ Rheumatol 31: 1659-1662. [Crossref]

6. Byers PD, Cotton RE, Deacon OW, Lowy M, Newman PH, et al. (1968) The diagnosis and treatment of pigmented villonodular synovitis. J Bone Joint Surg Br 50: 290-305. [Crossref]

7. Cheng XG, You YH, Liu W, Zhao T, Qu H (2004) MRI features of pigmented villonodular synovitis (PVNS). Clin Rheumatol 23: 31-34. [Crossref]

8. Hughes TH, Sartoris DJ, Schweitzer ME, Resnick DL (1995) Pigmented villonodular synovitis: MRI characteristics. Skeletal Radiol 24: 7-12. [Crossref]

9. Muscolo DL, Makino A, Costa-Panz M. Ayerza MA (1995) Localized pigmented villonodular synovitis of the posterior compartment of the knee: diagnosis with magnetic resonance imaging. Arthroscopy 11: 482-485.

10. Johansson JE, Ajjoub S, Coughlin LP, Wener JA, Cruess RL (1982) Pigmented villonodular synovitis of joints. Clin Orthop Relat Res: 159-166. [Crossref]

11. Kim SJ, Shin SJ, Choi NH, Choo ET (2000) Arthroscopic treatment for localized pigmented villonodular synovitis of the knee. Clin Orthop Relat Res: 224-230. [Crossref]

12. Beguin J, Locker B, Vielpeau C, Souquieres G (1989) Pigmented villonodular synovitis of the knee: results from 13 cases. Arthroscopy 5: 62-64. [Crossref]

13. Verspoor FG, Zee AA, Hannink G, van der Geest IC, Veth RP, et al. (2014) Longterm follow-up results of primary and recurrent pigmented villonodular synovitis Rheumatology 53: 2063-2070.

14. Aurégan JC, Klouche S, Bohu Y, Lefčvre N, Herman S, et al. (2014) Treatment of pigmented villonodular synovitis of the knee. Arthroscopy 30: 1327-1341. [Crossref]

Copyright: (C2016 Choi S. This is an open-access article distributed under the terms of the Creative Commons Attribution License, which permits unrestricted use, distribution, and reproduction in any medium, provided the original author and source are credited. 